\title{
Identifying disordered eating behaviours in adolescents: how do parent and adolescent reports differ by sex and age?
}

\author{
Savani Bartholdy ${ }^{1}$ (i) $\cdot$ Karina Allen $^{2} \cdot$ John Hodsoll $^{3} \cdot$ Owen G. O'Daly $^{4} \cdot$ Iain C. Campbell $^{1} \cdot$ Tobias Banaschewski $^{5}$. \\ Arun L. W. Bokde ${ }^{6}$ Uli Bromberg $^{7} \cdot$ Christian Büchel $^{7}$ - Erin Burke Quinlan ${ }^{8}$ - Patricia J. Conrod ${ }^{9,10}$ . \\ Sylvane Desrivières ${ }^{8} \cdot$ Herta Flor $^{11}$ • Vincent Frouin ${ }^{12} \cdot$ Jürgen Gallinat $^{13} \cdot$ Hugh Garavan $^{14} \cdot$ Andreas Heinz $^{15}$. \\ Bernd Ittermann $^{16}$ - Jean-Luc Martinot ${ }^{17,18} \cdot$ Eric Artiges $^{17,19} \cdot$ Frauke Nees $^{5,11} \cdot$ Dimitri Papadopoulos Orfanos $^{12}$. \\ Tomáš Paus $^{20} \cdot$ Luise Poustka ${ }^{5,21} \cdot$ Michael N. Smolka ${ }^{22} \cdot$ Eva Mennigen $^{22} \cdot$ Henrik Walter $^{15} \cdot$ Robert Whelan $^{23}$. \\ Gunter Schumann $^{8} \cdot$ Ulrike Schmidt $^{1,2}$
}

Received: 9 August 2016 / Accepted: 19 December 2016 / Published online: 3 January 2017

(C) The Author(s) 2017. This article is published with open access at Springerlink.com

\begin{abstract}
This study investigated the prevalence of disordered eating cognitions and behaviours across mid-adolescence in a large European sample, and explored the extent to which prevalence ratings were affected by informant (parent/adolescent), or the sex or age of the adolescent. The Development and Well-Being Assessment was completed by parent-adolescent dyads at age $14(n=2225)$ and again at age $16(n=1607)$ to explore the prevalence of 7 eating disorder symptoms (binge eating, purging, fear of weight gain, distress over shape/weight, avoidance of fattening foods, food restriction, and exercise for weight loss). Informant agreement was assessed using kappa
\end{abstract}

Savani Bartholdy

savani.bartholdy@kcl.ac.uk

1 Section of Eating Disorders, Department of Psychological Medicine, Institute of Psychiatry, Psychology and Neuroscience, King's College London, London, UK

2 South London and Maudsley NHS Foundation Trust, London, UK

3 Department of Biostatistics, Institute of Psychiatry, Psychology and Neuroscience, King's College London, London, UK

4 Centre for Neuroimaging Sciences, Institute of Psychiatry, Psychology and Neuroscience, King's College London, London, UK

5 Department of Child and Adolescent Psychiatry and Psychotherapy, Central Institute of Mental Health, Medical Faculty Mannheim, Heidelberg University, Square J5, 68159 Mannheim, Germany

6 Discipline of Psychiatry, School of Medicine and Trinity College Institute of Neuroscience, Trinity College Dublin, Dublin, Ireland

7 University Medical Centre Hamburg-Eppendorf, House W34, 3.OG, Martinistr. 52, 20246 Hamburg, Germany coefficients. Generalised estimating equations were performed to explore the impact of age, sex and informant on symptom prevalence. Slight to fair agreement was observed between parent and adolescent reports (kappa estimates between 0.045 and 0.318 ); however, this was largely driven by agreement on the absence of behaviours. Disordered eating behaviours were more consistently endorsed amongst girls compared to boys (odds ratios: 2.96-5.90) and by adolescents compared to their parents (odds ratios: 2.71-9.05). Our data are consistent with previous findings in epidemiological studies. The findings suggest that sex-related differences in the prevalence of disordered eating behaviour
8 Medical Research Council-Social, Genetic and Developmental Psychiatry Centre, Institute of Psychiatry, Psychology and Neuroscience, King's College London, London, UK

9 Department of Psychiatry, Université de Montréal, CHU Ste Justine Hospital, Quebec, Canada

10 Department of Psychological Medicine and Psychiatry, Institute of Psychiatry, Psychology and Neuroscience, King's College London, London, UK

11 Department of Cognitive and Clinical Neuroscience, Central Institute of Mental Health, Medical Faculty Mannheim, Heidelberg University, Square J5, Mannheim, Germany

12 Neurospin, Commissariat à l'Energie Atomique, CEA-Saclay Center, Paris, France

13 Department of Psychiatry and Psychotherapy, University Medical Center Hamburg-Eppendorf (UKE), Martinistrasse 52, 20246 Hamburg, Germany

14 Departments of Psychiatry and Psychology, University of Vermont, Burlington, VT 05405, USA

15 Department of Psychiatry and Psychotherapy, Campus Charité Mitte, Charité, Universitätsmedizin Berlin, Charitéplatz 1, Berlin, Germany 
are established by mid-adolescence. The greater prevalence rates obtained from adolescent compared to parent reports may be due to the secretive nature of the behaviours and/ or lack of awareness by parents. If adolescent reports are overlooked, the disordered behaviour may have a greater opportunity to become more entrenched.

Keywords Parent $\cdot$ Adolescent $\cdot$ Epidemiology $\cdot$ Eating disorders

\section{Introduction}

Eating disorders (EDs) are characterised by pathological concerns over shape and weight, and disturbed eating and weight-control behaviour. They are more common in females and typically start during adolescence, with a peak onset between ages 15 and 20 [1-3]. However, the age at which disordered eating behaviours (DEBs) and associated cognitions initially develop has not been widely studied, and it is unclear at what age the sex differences in the prevalence of DEBs emerge. Large prospective longitudinal cohort studies of community-dwelling adolescents are required to answer such questions, although the optimal method of assessing DEBs in adolescents remains unclear.

The use of multiple informants in assessing emotional and behavioural problems in youth is often advocated [4], as multiple perspectives of a child's behaviour are likely to enrich assessment, and can be important in diagnosing disorders involving symptom denial [5]. It has been proposed that parental reports may be beneficial for assessing

16 Physikalisch-Technische Bundesanstalt (PTB), Abbestr. 2-12, Berlin, Germany

17 Institut National de la Santé et de la Recherche Médicale, INSERM Unit 1000 "Neuroimaging \& Psychiatry", University Paris Sud, University Paris Descartes-Sorbonne Paris Cité, Paris, France

18 Maison de Solenn, Paris, France

19 Psychiatry Department 91G16, Orsay Hospital, Orsay, France

20 Rotman Research Institute, Baycrest and Departments of Psychology and Psychiatry, University of Toronto, Toronto, ON M6A 2E1, Canada

21 Department of Child and Adolescent Psychiatry and Psychotherapy, Medical University of Vienna, Vienna, Austria

22 Department of Psychiatry and Neuroimaging Center, Technische Universität Dresden, Dresden, Germany

23 Department of Psychology, University College Dublin, Dublin, Ireland anorexia nervosa (AN) as sufferers themselves often downplay symptoms at the start of the illness [6]. In contrast, parents may be unaware and under-report behaviours characteristic of bulimia nervosa (BN) that are often associated wtih secrecy and shame, such as binge eating and purging [5]. However, agreement between informants tends to be low $[4,7]$. Moreover, reliance on multiple informants may be problematic, as others' responses may be biased by their own attitudes, personality and internal state [8-10].

Poor-to-moderate agreement between youth and parent ratings has been observed for DEB among clinical samples. For example, Mariano et al. [11] found acceptable agreement for the presence of behavioural symptoms (e.g. binge eating, self-induced vomiting, and laxative/diuretic misuse), but poor agreement on frequency of behaviours and experience of disordered eating cognitions, with greater severity reported by young people compared to their parents. Similarly, Salbach-Andrae et al. [12] observed poor concordance between parent and adolescent reports, particularly for internalising behaviours. While several studies have observed good concordance for symptoms of AN [11], one study reported less concerns over weight and restraint in child reports (aged 6-12 years) compared to their parents [6], and another study revealed greater concordance for adolescents with BN compared to those with AN-Restrictive subtype [12]. In contrast, youths suffering from $\mathrm{BN}$ have been found to report greater severity of cognitions and frequency of behaviours [11], shape concerns and restraint [6] compared to their parents. Thus, concordance between parent and youth reports in clinical populations may depend on the nature of the behaviour and the stage or severity of illness.

Similar levels of non-concordance have been reported in non-clinical samples. Studies have reported good agreement on the absence of DEBs and modest agreement for the presence of eating disordered cognitions [13], but poor concordance for bulimic symptoms such as binge eating $[13,14]$. One study observed that similar prevalences of DEBs were reported by parents and youth, but found high levels of within-dyad disagreement [5]. Thus, parents may not be aware of their children's engagement in such behaviours [15]. Additionally, parents and children may differ in their understanding of problematic eating behaviours [5]. It is, therefore, important to assess parent-child agreement on both behavioural and cognitive symptoms to understand how best to identify symptoms amongst young people at a high-risk age (early-mid-adolescence) in the community.

Moreover, there has been little research into factors that affect concordance between youth and parental reports. Only one study has explored the degree to which informant (adolescents and their mothers) and sex influence the prevalence of DEBs in a large UK community sample [5]. The present study aims to (a) characterise the point prevalence 
of DEBs at ages 14 and 16 in a large multinational community sample based on adolescent self-reports and parental reports [IMAGEN cohort; 16], and (b) explore the concordance between parent and youth ratings of DEBs. This study extends the assessment of parent-youth agreement to a large multinational European cohort to explore the generalisability of findings across cultures. We predict that prevalence of DEBs will be higher in later adolescence (at age 16 compared to 14) and in girls at both ages compared to boys. Based on previous studies assessing multipleinformant agreement on DEBs in non-clinical samples, we hypothesise that greater agreement would be observed on disordered eating cognitions (fear of weight gain, distress over shape and weight), and behaviours (avoidance of fattening foods, food restriction, and exercise for weight loss) compared to binge eating and purging, which are additionally predicted to be more frequently endorsed by adolescent self-reports compared to parent reports, given the secretive nature of these behaviours.

\section{Materials and methods}

\section{Participants}

Participants were those taking part in a large multinational cohort study [the IMAGEN study: for further details, see 16]. Participants at age 14 (time point 1; T1) and their parents were recruited from secondary schools in 8 sites across the UK, Ireland, France and Germany. A total of 2225 parent-adolescent dyads completed the Development and Well-Being Assessment (DAWBA) online at T1; however, only 2215 of these pairs had data from the Dieting, Weight and Body Shape section (assessing ED symptoms) from at least 1 informant [43 dyads had data from only 1 informant (21 dyads with adolescent data only)]. 1607 parent-adolescent dyads also completed the DAWBA when the adolescent was aged 16 (time point 2; T2) (including an additional 2 pairs with incomplete baseline data); however, only 1604 pairs had data from the Dieting, Weight and Body Shape section from at least 1 informant (53 dyads with data from only 1 informant [ 25 dyads with adolescent data only]).

\section{Measures}

\section{DAWBA interview}

The DAWBA is a semi-structured interview that assesses the presence and frequency of symptoms of a number of psychiatric disorders. A youth and a parent version of the DAWBA were administered. Following on from a previous study exploring EDs in early adolescence using the
DAWBA interview [17], parent-adolescent agreement on the presence/absence of seven specific symptoms within the preceding 3 months was assessed: fear of weight gain (question 8), distress about shape/weight (question 11), avoidance of fattening foods (question 26), food restriction [composite measure including skipping meals (question 18a), eating less at meals (question 18b) and fasting (question 18c)], exercising for weight loss (question 18e), binge eating (eating an objectively large amount of food with associated loss of control; questions 15 and 16) and purging (actively getting rid of ingested food by self-induced vomiting or pill use; questions $1 \mathrm{c}, 18 \mathrm{f}$ and $18 \mathrm{~g}$ ).

\section{Body mass index}

In adolescents, body mass index (BMI: $\mathrm{kg} / \mathrm{m}^{2}$ ) is dependent on age and sex [18]. BMI z-scores were calculated based on the Centre for Disease Control and Prevention (CDC) Growth Charts correcting for age (in months) and sex [19] to determine how an individual's weight-for-height compares to children of the same age and sex using an external reference standard [18]. Following CDC recommendations, the following cutoffs were used: $>=95$ th percentile for obesity, 85th-95th percentile for overweight, and $<5$ th percentile for underweight.

\section{Procedure}

Interview, questionnaire, genetic and neuroimaging data were obtained from adolescents at age 14 (T1), and interview and questionnaire data were obtained online two years later at age 16 (T2). Interview and questionnaire data were collected from the parents at both time points. Only the responses on the Dieting, Weight and Body Shape section of the Development and Well-Being Assessment (DAWBA) interview were used here. The DAWBA interview was administered online, and height and weight were measured in person. Procedures were approved by the local ethics committees at each site, and were conducted in accordance with the Declaration of Helsinki. Written informed consent from the parents and written assent from the children were obtained prior to participation.

\section{Statistical analysis}

The DAWBA assesses presence and severity using an ordinal scale, providing the following options: "no", "a little", "a lot" or "tries but not allowed". Only participants that responded to the screening questions of the DAWBA Dieting, Weight and Body Shape section were included in the analyses. Responses were dichotomised into presence/ absence ratings: consistent ratings of "no" on the section's screening questions (due to the use of skip rules) or a rating 
of "no" on the specific symptom question(s) were marked as the DEB being absent, and any of the other ratings were marked as the DEB being present. For binge eating and purging behaviours, one question only assessed presence or absence without assessing severity; however, if the presence of either behaviour was indicated, the participant was included in the counts for these behaviours. Hence, some participants may have reported engaging in binge eating or purging behaviour without providing a frequency rating.

The presence/absence ratings were entered into a $2 \times 2$ contingency table for each DEB at each time point. Agreement between parent and adolescent ratings was assessed using the kappa coefficient. Values for kappa coefficients range between 0 and $1(\leq 0=$ poor, $0.01-0.20=$ slight, $0.21-0.40=$ fair, $0.41-0.60=$ moderate, $0.61-0.80=$ substantial, $0.81-1=$ almost perfect [20]). Symptom prevalence was first explored by calculating the percentage of adolescents for whom the symptom was endorsed by adolescent self-report, parent report, or either informant (using the OR rule). Secondly, we explored parental agreement for children who reported any behaviour at both time points, to establish whether parental agreement increased over time with respect to the adolescents who continued to perceive themselves to be symptomatic, and vice versa. Finally, we modelled the main effects of informant (parent/child), sex (boys/girls) and age (14/16) as predictors for each symptom using generalised estimating equations (GEE). GEE was employed over generalised linear mixed models, as we were interested in the impact of informant, sex and age as predictors at the population level, rather than at a subject-specific level (i.e. population-averaged odds rather than subject-specific odds [21]). As this analysis included within-subjects factors, independence could not be assumed, thereby prohibiting simple logistic regressions. GEE models assume that cases are not independent, assume a correlation between errors (the structure of which are specified by a covariance matrix) and do not assume heterogeneity of variance; thus, the GEE approach can be used to analyse non-normal within-subject data [22]. The GEE employed a binary logistic model with a logit link and an exchangeable working correlation matrix. As our analysis included two time points, the exchangeable and firstorder autoregressive working correlation matrices would be expected to produce roughly similar results [23]. Previous studies have also reported similar estimates and standard errors using exchangeable, independent and unspecified working correlation matrices on data assessed at two time points [21]. To deal with missing data, multiple imputation based on fully conditional specification was performed to allow data to be missing at random (MAR). Although GEE models are typically taken to allow data to be missing completely at random, Satty et al. [24] show that GEE with multiple imputation can perform well under the assumption of MAR, conditional on the important predictors of missingness being included in the model. Here, age, sex and informant were included as predictors of missingness. All models were run on 100 imputed datasets, and the presented are pooled estimates combined using Rubin's rules. All analyses were conducted in IBM SPSS Statistics version 21.

\section{Results}

\section{Descriptive statistics}

Demographic information is shown in Table 1. An approximately equal proportion of boys and girls participated at both time points. Most of the parental reports were provided by the mothers, followed by the fathers. The remaining reports were provided by both parents together, or by caregivers or guardians such as step- or foster parents, other relatives or a residential care worker.

\section{Three-month prevalence of DEB}

The prevalence of the seven DEBs was stratified by sex and informant (adolescent, parent or either informant [5]) at both time points (Table 2): prevalence was based on the total number of participants providing a presence/absence rating for each DEB. All DEBs were endorsed to a greater extent in adolescent reports compared to parent reports. Prevalences were highest when endorsement by either the parent or child was taken into account. However, there was only a small difference in prevalence estimates between adolescent reports and endorsements using either informant, whereas there was a notable difference in prevalence between either informant and parent reports. Similar prevalences were reported at both time points across informants. Of note, the prevalence of binge eating and purging almost doubled from age 14 to age 16 from adolescent reports, whereas the prevalence of binge eating decreased over time in parent reports. Across all informant types and DEBs, there was greater endorsement in girls compared to boys. Concordance was driven by agreement on the absence of the behaviour. Kappa estimates were between 0.045 and 0.318 , suggesting at most slight to fair agreement. Given the large prevalence index (i.e. the difference between the number of dyads agreeing on the presence compared to the absence of the behaviour), the proportion of chance agreement is expected to be high, which may have contributed to the low kappa estimates [25]. 
Table 1 Participant demographics

\begin{tabular}{|c|c|c|c|c|c|c|c|c|}
\hline & \multicolumn{4}{|l|}{ T1 (age 14) } & \multicolumn{4}{|l|}{ T2 (age 16) } \\
\hline & Girls & Boys & $\operatorname{Missing}^{\mathrm{a}}(n)$ & Total & Girls & Boys & $\operatorname{Missing}^{\mathrm{a}}(n)$ & Total \\
\hline Total $n$ & 1124 & 1072 & 29 & 2225 & 826 & 769 & 12 & 1607 \\
\hline Mean (SD) age (years) & $14.55(0.45)$ & $14.53(0.47)$ & $108(46 \mathrm{f})$ & $14.54(0.46)$ & $16.50(0.59)$ & $16.48(0.638)$ & $87(42 \mathrm{f})$ & $16.49(0.61)$ \\
\hline $\begin{array}{l}\text { Mean (SD) BMI ( } z \text {-score } \\
\text { adjusted for age and } \\
\text { sex) }\end{array}$ & $0.28(1.008)$ & $0.25(1.087)$ & 204 (111 f) & $0.26(1.048)$ & $0.16(1.305)$ & $0.31(1.435)$ & $444(195 \mathrm{f})$ & $0.23(1.367)$ \\
\hline \multicolumn{9}{|l|}{ Parent type $[n(\%)]^{b}$} \\
\hline Parent (unspecified) & $25[2.2 \%]$ & $19[1.8 \%]$ & $2[12.9 \%]$ & $46[2.2 \%]$ & $46[5.6 \%]$ & $45[5.9 \%]$ & $2[16.7 \%]$ & $93[5.8 \%]$ \\
\hline Mother & $881[78.5 \%]$ & $817[76.2 \%]$ & $17[54.8 \%]$ & $1715[77.1 \%]$ & $625[75.7 \%]$ & $571[74.3 \%]$ & $8[66.7 \%]$ & $1204[74.9 \%]$ \\
\hline Father & $181[16.1 \%]$ & 207 [19.3\%] & $6[19.4 \%]$ & $394[17.7 \%]$ & $122[14.8 \%]$ & $118[15.3 \%]$ & $2[16.7 \%]$ & $242[15.1 \%]$ \\
\hline Both parents & $4[0.4 \%]$ & $8[0.7 \%]$ & $0[0.0 \%]$ & $12[0.5 \%]$ & $5[0.6 \%]$ & $4[0.5 \%]$ & $0[0.0 \%]$ & $9[0.6 \%]$ \\
\hline Other caregiver type & $17[1.5 \%]$ & $10[0.9 \%]$ & $0[0.0 \%]$ & $27[1.2 \%]$ & $6[0.7 \%]$ & $5[0.7 \%]$ & $0[0.0 \%]$ & $11[0.7 \%]$ \\
\hline Missing & $14[1.2 \%]$ & $11[1.0 \%]$ & $4[12.9 \%]$ & $29[1.3 \%]$ & $22[2.7 \%]$ & $26[3.4 \%]$ & $0[0.0 \%]$ & $48[3.0 \%]$ \\
\hline \multicolumn{9}{|l|}{ Site $[n,(\%)]$} \\
\hline London & $147[13.1 \%]$ & $126[11.8 \%]$ & $0[0.0 \%]$ & $273[12.3 \%]$ & $134[16.2 \%]$ & 106 [13.8\%] & $0[0.0 \%]$ & 240 [14.9\%] \\
\hline Nottingham & 175 [15.6\%] & $185[17.3 \%]$ & $5[16.1 \%]$ & 365 [16.4\%] & 145 [17.6\%] & 138 [17.9\%] & $2[16.7 \%]$ & 285 [17.7\%] \\
\hline Dublin & $102[9.1 \%]$ & 118 [11.0\%] & $21[67.7 \%]$ & $241[10.8 \%]$ & 85 [10.3\%] & 96 [12.5\%] & 10 [83.3\%] & 191 [11.9\%] \\
\hline Paris & 130 [11.6\%] & $133[12.4 \%]$ & $1[3.2 \%]$ & 264 [11.9\%] & $72[8.7 \%]$ & $66[8.6 \%]$ & $0[0.0 \%]$ & $138[8.6 \%]$ \\
\hline Berlin & $144[12.8 \%]$ & $128[11.9 \%]$ & $2[6.5 \%]$ & $274[12.3 \%]$ & 80 [9.7\%] & $56[7.3 \%]$ & $0[0.0 \%]$ & $136[8.5 \%]$ \\
\hline Hamburg & $145[12.9 \%]$ & $121[11.3 \%]$ & $0[0.0 \%]$ & $265[11.9 \%]$ & 107 [13.0\%] & 99 [12.9\%] & $0[0.0 \%]$ & 206 [12.8\%] \\
\hline Mannheim & 153 [13.6\%] & $122[11.4 \%]$ & $2[6.5 \%]$ & 277 [12.4\%] & 105 [12.7\%] & 96 [12.5\%] & $0[0.0 \%]$ & $201[12.5 \%]$ \\
\hline Dresden & $126[11.2 \%]$ & 139 [13.0\%] & $0[0.0 \%]$ & 265 [11.9\%] & 98 [11.9\%] & 112 [14.6\%] & 0 [0.0\%] & 210 [13.1\%] \\
\hline
\end{tabular}

${ }^{\text {a }}$ Number of participants with unknown sex. For rows referring to age and BMI, frequencies correspond to number of participants with missing age/BMI data

b Relative or guardian that provided parent responses on the DAWBA

\section{Stability of symptoms and changes in informant reports}

To explore whether concordance improved over time if the symptoms persisted, parental reports were assessed specifically for adolescents who reported the presence of any symptom at both time points and vice versa (Table 3).

In a combined assessment of bulimic behaviours, 83 adolescents endorsed binge eating and/or purging behaviours at both time points, whereas only 5 parents reported these behaviours at both time points. For only 3 of these parent-adolescent dyads, at least one of these behaviours was reported at both time points by both informants. Of the adolescents who endorsed bulimic behaviour(s) at both time points, 4 parents reported the behaviour at age 14 [binge eating $(n=3)$, binge eating and purging $(n=1)$ ], whereas at age 16 , the behaviours were reported by 8 parents [binge eating $(n=2)$, purging $(n=5)$, binge eating and purging $(n=1)]$. Of the parents who reported least one of these behaviours at both time points, all adolescents reported the behaviour at one or more time points:
1 reported the bingeing at $\mathrm{T} 1$ only, 1 reported purging at T2 only, and 3 reported binge eating/purging at both time points ( 2 reported both behaviours, 1 reported binge eating at $\mathrm{T} 1$ and purging at $\mathrm{T} 2$ ).

\section{Modelling prevalence based on informant and the adolescent's sex and age}

Table 4 presents the estimated prevalence of each symptom predicted by the informant, the adolescent's sex and the adolescent's age. The odds for the behaviour being endorsed by girls compared to boys, adolescents compared to parents, and at age 14 compared to 16 were determined by calculating the exponentiated beta coefficient (odds ratio; OR).

Girls were 2.96-5.90 times more likely to endorse any of the DEBs than boys. With the exception of distress over weight/shape, the differences in predicted prevalence of DEBs between age 14 and 16 were small yet statistically significant, though the direction of the differences was inconsistent. The prevalence of fear of weight gain, 
Table 2 Prevalence of disordered eating behaviours according to adolescent report, parent report, or either informant report (using "OR rule" method), and percentage agreement

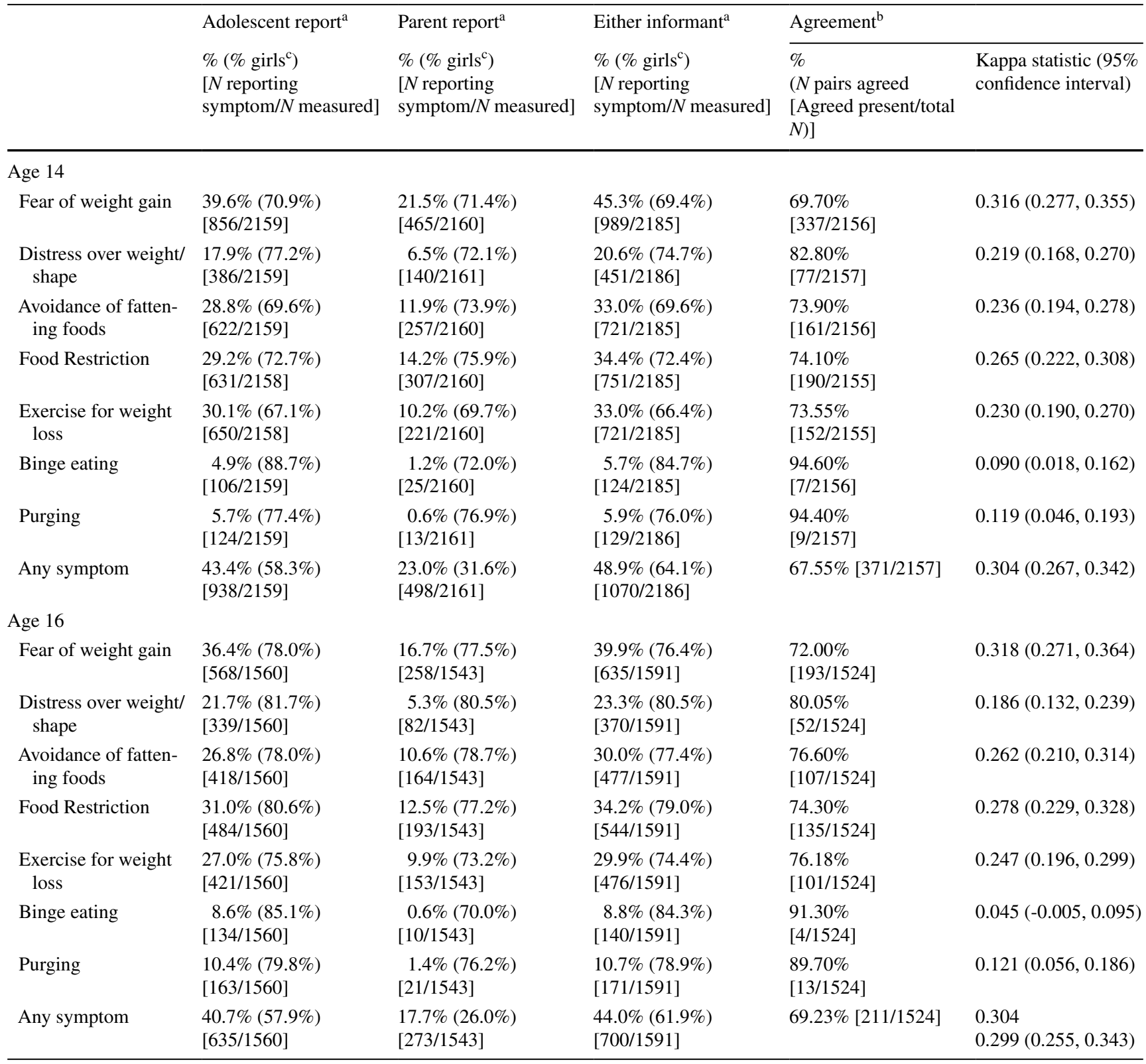

a Only included individuals with adolescent sex reported

b Included all individuals (regardless of whether sex was reported/missing)

c $\%$ of the sample reporting the symptom who were girls

avoidance of fattening foods, food restriction and exercise for weight loss were 1.07-1.34 times higher at age 14 than at age 16 , whereas binge eating and purging were $1.47-$ 1.88 times more prevalent at age 16 than 14 . Finally, adolescents were at least 2.71 times more likely to report any DEB than their parent. This was especially true for bulimic behaviours: adolescents were 7.44 times more likely to report binge eating and 9.05 times more likely to endorse purging compared to parent reports.

\section{Discussion}

This study assessed the three-month prevalence of DEB across mid-adolescence in a multinational European sample based on adolescent and parent reports, and investigated the degree of concordance between informants. It was predicted that DEBs would be more prevalent amongst girls compared to boys, adolescent reports compared to parents, and at age 16 compared to age 14 . 


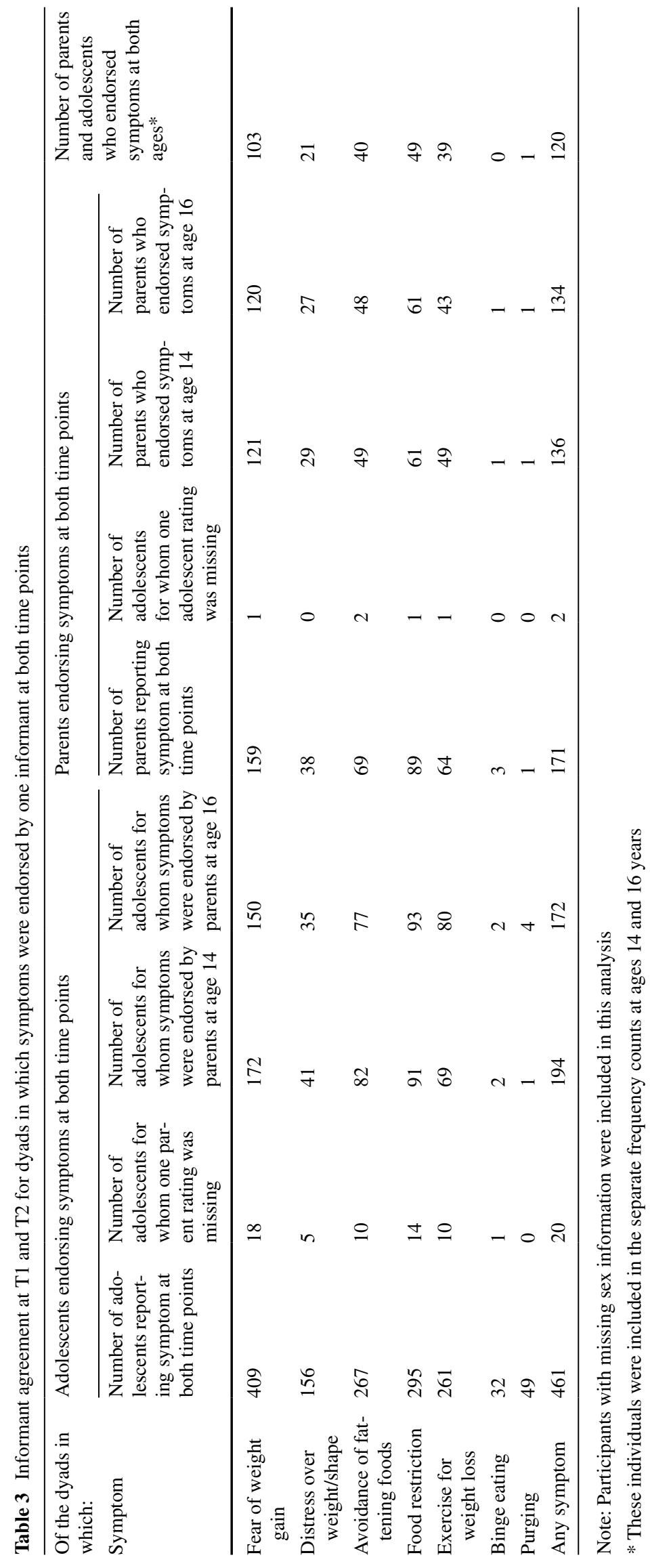


Table 4 Modelling prevalence by adolescent's age, adolescent's sex and informant: Odd's ratios (OR) [95\% confidence intervals (CI)] calculated using each sex, informant and time point as the reference category

\begin{tabular}{|c|c|c|c|c|c|c|c|c|c|}
\hline \multirow{2}{*}{$\begin{array}{l}\text { Symptom } \\
\text { (Reference } \\
\text { category) }\end{array}$} & \multicolumn{3}{|l|}{ Sex } & \multicolumn{3}{|l|}{ Informant } & \multicolumn{3}{|l|}{ Age } \\
\hline & (Girls) & (Boys) & $p$ & (Adolescent) & (Parent) & $p$ & (T1) & $(\mathrm{T} 2)$ & $p$ \\
\hline \multicolumn{10}{|c|}{ Fear of weight gain } \\
\hline OR $(95 \% \mathrm{CI})$ & $\begin{array}{l}0.241(.209, \\
.279)\end{array}$ & $\begin{array}{l}4.145(3.585 \\
4.793)\end{array}$ & $<0.001$ & $\begin{array}{c}0.369(.331 \\
.411)\end{array}$ & $\begin{array}{l}2.711(2.435 \\
3.017)\end{array}$ & $<0.001$ & $\begin{array}{l}0.744(.674 \\
.821)\end{array}$ & $\begin{array}{l}1.344(1.217 \\
1.484)\end{array}$ & $<0.001$ \\
\hline \multicolumn{10}{|c|}{ Distress over weight/shape } \\
\hline OR $(95 \% \mathrm{CI})$ & $\begin{array}{l}0.230(.189 \\
.280)\end{array}$ & $\begin{array}{l}4.344(3.569 \\
5.287)\end{array}$ & $<0.001$ & $\begin{array}{l}0.252(.216 \\
.295)\end{array}$ & $\begin{array}{c}3.963(4.636, \\
3.388)\end{array}$ & $<0.001$ & $\begin{array}{l}1.057(.930, \\
1.202)\end{array}$ & $\begin{array}{c}0.946(.832 \\
1.075)\end{array}$ & 0.393 \\
\hline \multicolumn{10}{|c|}{ Avoidance of fattening foods } \\
\hline OR $(95 \% \mathrm{CI})$ & $\begin{array}{l}0.285(.242 \text {, } \\
.334)\end{array}$ & $\begin{array}{l}3.514(2.990 \text {, } \\
4.131)\end{array}$ & $<0.001$ & $\begin{array}{l}0.316(.280 \\
.356)\end{array}$ & $\begin{array}{l}3.169(2.810 \\
3.573)\end{array}$ & $<0.001$ & $\begin{array}{l}0.823(.738, \\
.918)\end{array}$ & $\begin{array}{l}1.214(1.090 \\
1.354)\end{array}$ & $<0.001$ \\
\hline \multicolumn{10}{|l|}{ Food restriction } \\
\hline OR $(95 \% \mathrm{CI})$ & $\begin{array}{l}0.232(.198, \\
.273)\end{array}$ & $\begin{array}{l}4.306(3.669 \text {, } \\
5.054)\end{array}$ & $<0.001$ & $\begin{array}{l}0.344(.306, \\
.387)\end{array}$ & $\begin{array}{l}2.907(2.583 \text {, } \\
3.272)\end{array}$ & $<0.001$ & $\begin{array}{l}0.934(.837 \\
1.042)\end{array}$ & $\begin{array}{l}1.071(.960, \\
1.945)\end{array}$ & $<0.001$ \\
\hline \multicolumn{10}{|c|}{ Exercise for weight loss } \\
\hline OR $(95 \% \mathrm{CI})$ & $\begin{array}{l}0.338(.289 \\
.395)\end{array}$ & $\begin{array}{l}2.962(2.533, \\
3.464)\end{array}$ & $<0.001$ & $\begin{array}{l}0.269(.237, \\
.304)\end{array}$ & $\begin{array}{l}3.722(3.284 \\
4.217)\end{array}$ & $<0.001$ & $\begin{array}{l}0.833(.746, \\
.930)\end{array}$ & $\begin{array}{l}1.200(1.075 \\
1.340)\end{array}$ & 0.001 \\
\hline \multicolumn{10}{|l|}{ Binge eating } \\
\hline OR $(95 \% \mathrm{CI})$ & $\begin{array}{l}0.169(.117, \\
.246)\end{array}$ & $\begin{array}{l}5.901(4.067, \\
8.561)\end{array}$ & $<0.001$ & $\begin{array}{l}0.134(.092, \\
.196)\end{array}$ & $\begin{array}{l}7.440(5.110 \\
10.832)\end{array}$ & $<0.001$ & $\begin{array}{l}1.469 \\
1.850)\end{array}$ & $\begin{array}{l}0.681(.541, \\
.857)\end{array}$ & 0.001 \\
\hline \multicolumn{10}{|l|}{ Purging } \\
\hline OR $(95 \% \mathrm{CI})$ & $\begin{array}{l}0.273(.202, \\
.369)\end{array}$ & $\begin{array}{c}3.667 \\
(2.712 .957)\end{array}$ & $<0.001$ & $\begin{array}{l}0.111(.078, \\
.160)\end{array}$ & $\begin{array}{l}9.049(6.371, \\
12.851)\end{array}$ & $<0.001$ & $\begin{array}{l}1.876(1.508, \\
2.332)\end{array}$ & $\begin{array}{l}0.533(0.663 \text {, } \\
0.429)\end{array}$ & $<0.001$ \\
\hline \multicolumn{10}{|l|}{ Any symptom } \\
\hline OR $(95 \% \mathrm{CI})$ & $\begin{array}{l}0.272(.235 \\
.315)\end{array}$ & $\begin{array}{l}3.680(3.178 \\
4.261)\end{array}$ & $<0.001$ & $\begin{array}{l}0.330(.298, \\
.365)\end{array}$ & $\begin{array}{l}3.030(2.738 \\
3.352)\end{array}$ & $<0.001$ & $\begin{array}{l}0.831(.906, \\
.762)\end{array}$ & $\begin{array}{l}1.204(1.104 \\
1.313)\end{array}$ & $<0.001$ \\
\hline
\end{tabular}

Estimated marginal means (EM) were converted into percentages to reflect the prevalence of the behaviour according to predictor variable. Coefficients were exponentiated to present odds ratios (OR). All models were run on imputed datasets. The parameters presented are pooled estimates combined using Rubin's rules

Our data revealed that DEBs were more consistently endorsed amongst girls compared to boys and adolescents compared to their parents. However, prevalence did not vary widely as a function of age. This may be due to similarities between ages 14 and 16 years in terms of physical development (i.e. post-pubertal) and environmental challenges. Greater differences in prevalence may be expected if comparisons are made between time points characterised by different environmental challenges and both physical and neural developmental stages, i.e. pre- versus postpuberty, or early versus late adolescence. For example, in the context of clinical diagnoses, Allen et al. [26] reported that the prevalence of EDs increased significantly from age 14 to ages 17 and 20 in females but only between ages 14 and 20 in males. Thus, future studies may wish to model the impact of the interaction between age and sex on symptom prevalence.

DEBs were more prevalent amongst girls compared to boys at both time points. Although the magnitude of the sex differences observed in this study is smaller than that reported in treatment-seeking samples, it is of similar magnitude to population-based studies in adolescents [5, 17, 27, 28]. However, there is some inconsistency regarding the direction and specificity of the differences in prevalences between sexes [29]. For example, one recent study in the UK found a greater prevalence of some DEBs in girls compared to boys using parental reports (including fear of weight gain, distress about weight/shape, food restriction and avoidance of fattening foods), but equivalent endorsement of binge eating and purging across the sexes (approx. 5 and $0.2 \%$, respectively) [17]. Another study reported a greater prevalence of binge eating amongst girls compared to boys in adolescent self-reports, but no sex-related differences in parent reports [5]. In contrast, we found a greater prevalence of all DEBs in girls (including binge eating and purging) in both the parent and adolescent reports, suggesting that the sex-related differences in prevalence are not simply a matter of the informant. However, factors such as issues in general recognition of DEBs in boys or willingness to report may have contributed to these findings. 
For example, Lee-Winn et al. [30] reported that although no sex differences were observed with respect to recurrent overeating, emotional aspects of binge eating (loss of control, distress) were more prevalent in girls than boys. These authors suggested that this may be due to emotional expression being seen as less socially acceptable amongst boys.

Consistent with previous studies, our data revealed slight to fair concordance between parent and adolescent reports of ED symptoms. All symptoms were more prevalent amongst adolescent self-reports compared to parent reports, particularly with respect to binge eating and purging. This may be due to the secrecy and shame often associated with these behaviours (Wilfley et al. [31]). We also found that the number of parents reporting binge eating and/or purging in adolescents who report themselves as symptomatic at both time points does not improve over time. However, contrary to Swanson et al.'s [5] findings of increased prevalence estimates for some DEBs (e.g. binge eating, fasting) DEBs obtained using the "OR" rule (i.e. reported by either informant) compared to reports from adolescents/children only, combining parent and adolescent reports in our study elicited prevalence estimates that were only slightly higher than those from the adolescent reports alone for all DEBs. In contrast, they were substantially higher than the prevalence estimates from parent reports. These findings suggest that parents are unaware of their children's endorsement of such behaviours [15]. The lack of awareness of adolescents' binge eating and purging in mid-adolescence may contribute to a central issue in EDs, namely difficulty in early recognition. If this is correct, it is important to educate parents, raise awareness of these symptoms and to take note of symptoms in late childhood and early adolescence. However, such discrepancies may also be explained by differences in attitudes towards, understanding or interpretation of DEBs [4, 5]. Future studies may wish to investigate the similarity of interpretations of behavioural definitions between informants to address issues of comparability between reports.

The main strength of this study is the use of a large, representative, multinational community sample with repeated measurements at 2 time points and a range of parental/caregiver figures. We considered a range of ED symptoms and how the persistence of symptoms over 2 years may influence adolescent-parent concordance. Although we did not take into account the contact time between parents and adolescents, which may influence the parent's awareness of the adolescent's behaviour, it is expected that our sample encapsulates a broad array of parental involvement. Moreover, the use of a community sample may explain the high rates of concordance for the absence of DEBs [13].

This study has some limitations. Firstly, due to the use of skip rules in the DAWBA, the majority of the "absent" responses were presumed absent based on negative responses to all five screening questions. Thus, individuals who may engage in these behaviours although they did not endorse the screening questions would not have been accurately represented. However, post hoc exploration of the endorsement of entry questions yielded similar findings to our GEE models, whereby entry questions were more frequently endorsed in girls compared to boys (by both informants) and by adolescents compared to parents (for both sexes), and this did not vary widely between time points: (adolescents: 58.0-58.5\% girls and 23.1-29.7\% boys; parents: $20.9-25.2 \%$ girls and 5.3-9.6\% boys). Secondly, there were insufficient data to investigate differences in symptom severity. Finally, parental weight and parental history of an ED were not assessed. Parents experiencing difficulties with eating or weight regulation may not be as good at noticing eating problems in their children [32]. Moreover, maternal and paternal BMI have been reported to be predictors of ED caseness at age 14 compared to healthy controls and psychiatric controls, respectively [33]. Therefore, parental weight and disordered eating should be included as predictor variables in future models of symptom prevalence.

Our findings suggest that adolescent reports (compared to parent reports) elicit greater prevalence of ED symptoms in mid-adolescence in the community. It is unclear, however, whether the behaviours are being over- or underreported by adolescents and parents, respectively. While the use of multiple informants can provide a broader picture of the adolescent's behaviour, they may also introduce errors with inaccurate reporting of behaviour. Given the limitations of self-report, alternative perspectives provided by a second informant may be useful in identifying potentially vulnerable individuals who may not identify themselves as symptomatic, or for conceptualising an adolescent's behaviour in different contexts. Indeed, there has been some support for the use of computerised diagnostic assessments such as the DAWBA that takes into consideration multiple informants' reports to calculate the probability of a child/ adolescent having an ED [34, 35]. However, a Swiss study of child and adolescent outpatients comparing the ICD-10 diagnoses provided by clinicians to those reached through expert review of DAWBA data revealed that although agreement between the DAWBA expert and clinician ratings was observed, this was largely driven by negative ratings of EDs [36].

In the context of diagnoses determined using information from multiple informants, the question remains as to how to prioritise or combine this information. While parent reports may be superior and more practical when assessing young children [37], it is likely that adolescents will be sufficiently aware of their own thoughts/behaviours, and thus may be a reliable source of information. Additionally, parent reports may be useful in determining symptom severity, 
which this study was unable to assess. For example, it has been suggested that persistent disagreement between informants could indicate a poorer prognosis compared to individuals for whom informant ratings consistently converge [38]. However, parental reports may be important with respect to the denial that often occurs at the start of the illness, particularly in AN [6]. Indeed, in the present study, 51 additional cases were identified by parental reports as endorsing any DEB at both time points (who were not also endorsed at both time points by adolescent reports); however, this is substantially smaller than the 341 additional cases identified by adolescent reports. To better understand the utility of multiple informants in identification of DEB in adolescents, future studies should aim to clarify how concordance rates differ throughout childhood and adolescence in relation to a broader array of symptoms, the factors that influence concordance (e.g. parental weight/eating difficulties) and how this relates to prognosis and the duration of untreated symptoms/ED.

Acknowledgements This work received support from the following sources: the European Union-funded FP6 Integrated Project IMAGEN (Reinforcement-related behaviour in normal brain function and psychopathology) (LSHM-CT- 2007-037286), the FP7 projects IMAGEMEND (602450; IMAging GEnetics for MENtal Disorders), AGGRESSOTYPE (602805) and MATRICS (603016), the Innovative Medicine Initiative Project EU-AIMS (115300-2), the Medical Research Council Grants "Developmental pathways into adolescent substance abuse" (93558) and Consortium on Vulnerability to Externalizing Disorders and Addictions [c-VEDA] (MR/N000390/1), the Swedish funding agencies VR, FORTE and FORMAS, the Medical Research Council and the Wellcome Trust (Behavioural and Clinical Neuroscience Institute, University of Cambridge), the National Institute for Health Research (NIHR) Biomedical Research Centre at South London and Maudsley NHS Foundation Trust and King's College London, the Bundesministerium für Bildung und Forschung (BMBF Grants 01GS08152; 01EV0711; eMED SysAlc 01ZX1311A; Forschungsnetz AERIAL), the Deutsche Forschungsgemeinschaft (DFG Grants SM 80/7-1, SM 80/7-2, SFB 940/1). Further support was provided by Grants from: ANR (project AF12-NEUR0008-01WM2NA, and ANR-12-SAMA-0004), the Fondation de France, the Fondation pour la Recherche Médicale, the Mission Interministérielle de Lutte-contre-les-Drogues-et-les-Conduites-Addictives (MILDECA), the Assistance-Publique-Hôpitaux-de-Paris and INSERM (interface Grant), Paris Sud University IDEX 2012; the National Institutes of Health, USA (Axon, Testosterone and Mental Health during Adolescence; RO1 MH085772-01A1), and by NIH Consortium Grant U54 EB020403, supported by a cross-NIH alliance that funds Big Data to Knowledge Centres of Excellence.

Savani Bartholdy is supported by a $\mathrm{PhD}$ studentship from the National Institute of Health Research (NIHR) Biomedical Research Centre (BRC) at South London and Maudsley National Health Service (NHS) Foundation Trust and King's College London (KCL). John Hodsoll, Iain Campbell and Ulrike Schmidt receive salary support from the NIHR Mental Health BRC at SLaM and KCL. Owen O'Daly receives salary support from an NIHR Infrastructure grant for the Wellcome Trust/KCL Clinical Research Facility. The views are those of the authors and not necessarily those of the NHS, the NIHR or the Department of Health.

\section{Compliance with ethical standards}

Ethical approval The study procedures were approved by the local ethics committee at each respective site, and were conducted in accordance with the Declaration of Helsinki. Written informed consent from the parents and written assent from the children were obtained prior to participation.

Conflict of interest Dr. Banaschewski has served as an advisor or consultant to Bristol-Myers Squibb, Desitin Arzneimittel, Eli Lilly, Medice, Novartis, Pfizer, Shire, UCB, and Vifor Pharma; he has received conference attendance support, conference support, or speaking fees from Eli Lilly, Janssen McNeil, Medice, Novartis, Shire, and UCB; and he is involved in clinical trials conducted by Eli Lilly, Novartis, and Shire; the present work is unrelated to these relationships. Dr. Gallinat has received research funding from the German Federal Ministry of Education and Research, AstraZeneca, Eli Lilly, Janssen-Cilag, and Bristol-Myers Squibb; he has received speaking fees from AstraZeneca, Janssen-Cilag, and Bristol-Myers Squibb. Dr Barker has received honoraria from General Electric for teaching on scanner programming courses. The other authors report no biomedical financial interests or potential conflicts of interest.

Open Access This article is distributed under the terms of the Creative Commons Attribution 4.0 International License (http://creativecommons.org/licenses/by/4.0/), which permits unrestricted use, distribution, and reproduction in any medium, provided you give appropriate credit to the original author(s) and the source, provide a link to the Creative Commons license, and indicate if changes were made.

\section{References}

1. Stice E, Marti CN, Rohde P (2013) Prevalence, incidence, impairment, and course of the proposed DSM- 5 eating disorder diagnoses in an 8-year prospective community study of young women. J Abnorm Psychol 122:445. doi:10.1037/a0030679

2. Micali N, Hagberg KW, Petersen I, Treasure JL (2013) The incidence of eating disorders in the UK in 2000-2009: findings from the General Practice Research Database. BMJ Open. doi:10.1016/s0140-6736(13)60515-2

3. Smink FR, van Hoeken D, Hoek HW (2012) Epidemiology of eating disorders: incidence, prevalence and mortality rates. Curr Psychiatry Rep 14:406-414. doi:10.1007/s11920-012-0282-y

4. deKeyser L, Svedin CG, Agnafors S, Bladh M, Sydsjö G (2014) Multi-informant reports of mental health in Swedish-born children of immigrants and children born to non-immigrants - the SESBiC-study. BMC Pediatr 14:1-9. doi:10.1186/1471-2431-14-95

5. Swanson SA, Aloisio KM, Horton NJ, Sonneville KR, Crosby RD, Eddy KT, Field AE, Micali N (2014) Assessing eating disorder symptoms in adolescence: is there a role for multiple informants? Int J Eat Disord 47:475-482. doi:10.1002/eat.22250

6. Couturier J, Lock J, Forsberg S, Vanderheyden D, Yen HL (2007) The addition of a parent and clinician component to the eating disorder examination for children and adolescents. Int J Eat Disord 40:472-475. doi:10.1002/eat.20379

7. Achenbach TM, McConaughy SH, Howell CT (1987) Child/ adolescent behavioral and emotional problems: implications of cross-informant correlations for situational specificity. Psychol Bull 101:213-232. doi:10.1037/0033-2909.101.2.213 
8. Zapolski TC, Smith GT (2013) Comparison of parent versus child-report of child impulsivity traits and prediction of outcome variables. J Psychopathol Behav Assess 35:301-313. doi:10.1007/s10862-013-9349-2

9. Klonsky ED, Oltmanns TF (2002) Informant-reports of personality disorder: relation to self-reports and future research directions. Clin Psychol Sci Prac 9:300-311. doi:10.1093/ clipsy.9.3.300

10. Müller JM, Achtergarde S, Furniss T (2011) The influence of maternal psychopathology on ratings of child psychiatric symptoms: an SEM analysis on cross-informant agreement. Eur Child Adolesc Psychiatry 20:241-252. doi:10.1007/ s00787-011-0168-2

11. Mariano P, Watson HJ, Leach DJ, McCormack J, Forbes DA (2013) Parent-child concordance in reporting of child eating disorder pathology as assessed by the eating disorder examination. Int J Eat Disord 46:617-625. doi:10.1002/eat.22158

12. Salbach-Andrae H, Klinkowski N, Lenz K, Pfeiffer E, Lehmkuhl U, Ehrlich S (2008) Correspondence between self-reported and parent-reported psychopathology in adolescents with eating disorders. Psychopathology 41:307-312. doi:10.1159/000146068

13. Steinberg E, Tanofsky-Kraff M, Cohen ML, Elberg J, Freedman RJ, Semega-Janneh M, Yanovski SZ, Yanovski JA (2004) Comparison of the child and parent forms of the Questionnaire on Eating and Weight Patterns in the assessment of children's eating-disordered behaviors. Int J Eat Disord 36:183-194. doi:10.1002/eat.20022

14. Johnson WG, Grieve FG, Adams CD, Sandy J (1999) Measuring binge eating in adolescents: adolescent and parent versions of the questionnaire of eating and weight patterns. Int $\mathrm{J}$ Eat Disord 26:301-314. doi:10.1002/ (SICI)1098-108X(199911)26:3<301:AID-EAT8>3.0.CO;2-M

15. Tanofsky-Kraff M, Yanovski SZ, Yanovski JA (2005) Comparison of child interview and parent reports of children's eating disordered behaviors. Eat Behav 6:95-99. doi:10.1002/eat.10128

16. Schumann G, Loth E, Banaschewski T, Barbot A, Barker G, Büchel C, Conrod P, Dalley J, Flor H, Gallinat J (2010) The IMAGEN study: reinforcement-related behaviour in normal brain function and psychopathology. Mol Psychiatry 15:11281139. doi: $10.1038 / \mathrm{mp} .2010 .4$

17. Micali N, Ploubidis G, De Stavola B, Simonoff E, Treasure J (2014) Frequency and patterns of eating disorder symptoms in early adolescence. J Adolesc Health 54:574-581. doi:10.1016/j. jadohealth.2013.10.200

18. Must A, Anderson S (2006) Pediatric mini review. Body mass index in children and adolescents: considerations for population-based applications. Int J Obes 30:590-594. doi:10.1038/ sj.ijo. 0803300

19. Centers for Disease Control and Prevention (2000) Percentile data files with LMS values. http://www.cdc.gov/growthcharts/ percentile_data_files.htm. Accessed 19 April 2016

20. Landis JR, Koch GG (1977) The measurement of observer agreement for categorical data. Biometrics 33:159-174. doi: $10.2307 / 2529310$

21. Hu FB, Goldberg J, Hedeker D, Flay BR, Pentz MA (1998) Comparison of population-averaged and subject-specific approaches for analyzing repeated binary outcomes. Am J Epidemiol 147:694-703. doi:10.1093/oxfordjournals.aje.a009511

22. Ballinger GA (2004) Using generalized estimating equations for longitudinal data analysis. Organ Res Methods 7:127-150. doi: $10.1177 / 1094428104263672$
23. Tang W, He H, Tu XM (2012) Applied categorical and count data analysis. CRC Press, Boca Raton

24. Satty A, Mwambi H, Molenberghs G (2014) Different methods for handling longitudinal binary outcome subject to potentially random dropout. Stat Methodol 24:12-27. doi:10.1016/j. stamet.2014.10.002

25. Viera AJ, Garrett JM (2005) Understanding interobserver agreement: the kappa statistic. Fam Med 37:360-363

26. Allen KL, Byrne SM, Oddy WH, Crosby RD (2013) DSM-IVTR and DSM-5 eating disorders in adolescents: prevalence, stability, and psychosocial correlates in a population-based sample of male and female adolescents. J Abnorm Psychol 122:720 732. doi: $10.1037 / \mathrm{a} 0034004$

27. Swanson SA, Crow SJ, Le Grange D, Swendsen J, Merikangas KR (2011) Prevalence and correlates of eating disorders in adolescents: results from the national comorbidity survey replication adolescent supplement. Arch Gen Psychiatry 68:714-723. doi:10.1001/archgenpsychiatry.2011.22

28. Smink FR, Hoeken D, Oldehinkel AJ, Hoek HW (2014) Prevalence and severity of DSM-5 eating disorders in a community cohort of adolescents. Int J Eat Disord 47:610-619. doi:10.1002/eat.22316

29. Striegel-Moore RH, Rosselli F, Perrin N, DeBar L, Wilson GT, May A, Kraemer HC (2009) Gender difference in the prevalence of eating disorder symptoms. Int J Eat Disord 42:471-474. doi:10.1002/eat.20625

30. Lee-Winn AE, Reinblatt SP, Mojtabai R, Mendelson T (2016) Gender and racial/ethnic differences in binge eating symptoms in a nationally representative sample of adolescents in the United States. Eat Behav 22:27-33. doi:10.1016/j.eatbeh.2016.03.021

31. Wilfley DE, Schwartz MB, Spurrell EB, Fairburn CG (1997) Assessing the specific psychopathology of binge eating disorder patients: Interview or self-report? Behav Res Ther 35:11511159. doi:10.1016/S0005-7967(97)80010-1

32. Boutelle K, Fulkerson JA, Neumark-Sztainer D, Story M (2004) Mothers' perceptions of their adolescents' weight status: are they accurate? Obes Res 12:1754-1757. doi:10.1038/oby.2004.217

33. Allen KL, Byrne SM, Forbes D, Oddy WH (2009) Risk factors for full- and partial-syndrome early adolescent eating disorders: a population-based pregnancy cohort study. J Am Acad Child Adolesc Psychiatry 48:800-809. doi:10.1097/CHI.0b013e3181a8136d

34. House J, Eisler I, Simic M, Micali N (2008) Diagnosing eating disorders in adolescents: a comparison of the eating disorder examination and the development and well-being assessment. Int J Eat Disord 41:535-541. doi:10.1002/eat.20528

35. Moya T, Fleitlich-Bilyk B, Goodman R, Nogueira FC, Focchi PS, Nicoletti M, Pinzon V, Cordás TA, Lotufo Neto F (2005) The eating disorders section of the Development and Well-Being Assessment (DAWBA): development and validation. Rev Bras Psyquiatr 27:25-31. doi:10.1590/s1516-44462005000100008

36. Aebi M, Kuhn C, Metzke CW, Stringaris A, Goodman R, Steinhausen H-C (2012) The use of the development and wellbeing assessment (DAWBA) in clinical practice: a randomized trial. Eur Child Adolesc Psychiatry 21:559-567. doi:10.1007/ s00787-012-0293-6

37. Braet C, Soetens B, Moens E, Mels S, Goossens L, Van Vlierberghe L (2007) Are two informants better than one? Parentchild agreement on the eating styles of children who are overweight. Eur Eat Disord Rev 15:410-417. doi:10.1002/erv.798

38. van der Ende J, Verhulst FC, Tiemeier H (2012) Agreement of informants on emotional and behavioral problems from childhood to adulthood. Psychol Assess 24:293. doi:10.1037/a0025500 